\title{
Cellular basis of defective sperm function and its association with the genesis of reactive oxygen species by human spermatozoa*
}

\author{
R. J. Aitken and J. S. Clarkson \\ MRC Reproductive Biology Unit, 37 Chalmers Street, Edinburgh EH3 9EW, U.K.
}

\begin{abstract}
Summary. Addition of the divalent cation ionophore, A23187, to washed populations of human spermatozoa resulted in a sudden burst of production of reactive oxygen species which peaked within 3-5 min. This activity was dependent upon the presence of calcium in the external medium and was unaffected by the mitochondrial inhibitors, oligomycin, antimycin and rotenone. Studies with scavengers of reactive oxygen species revealed that, while reagents directed against singlet oxygen and the hydroxyl radical were without effect, cytochrome $\mathrm{C}$ reduced the response to $\mathrm{A} 23187$ by about $50 \%$, suggesting that the superoxide anion radical is a major product of the activated human spermatozoon.

The clinical implications of these studies stem from the considerable variation observed between individuals in the levels of reactive oxygen species produced by the spermatozoa. This variability was shown to be inversely related to the ability of the spermatozoa to exhibit sperm-oocyte fusion on exposure to A23187; defective samples exhibited a basal level of reactive oxygen species production which was 40 times that observed with normal functional cells.
\end{abstract}

\section{Introduction}

Approximately 1 in 6 couples in the United Kingdom will have cause to visit a specialist infertility clinic during their reproductive life span (Hull, 1986). Within this population the most frequently encountered, defined condition is defective sperm function, which alone accounts for about $25 \%$ of all cases. In addition to these patients, studies using bioassays for sperm-oocyte fusion indicate that, in about one third of couples exhibiting idiopathic infertility, defects of sperm function can be detected, even though the semen profile is normal (Aitken et al., 1982). Despite this widespread occurrence of male infertility there are few, if any, effective treatments to correct this condition. This situation is a direct reflection of our poor state of knowledge concerning both the fundamental cell biology of the human spermatozoon and the biochemical nature of the lesions present in the spermatozoa of infertile patients.

However, recent progress in this area has been made following the introduction of the zona-free hamster oocyte penetration test to measure the competence of human spermatozoa to engage in sperm-oocyte fusion (Yanagimachi et al., 1976; Aitken, 1986). Such studies have demonstrated

*Presented as the Arthur Walpole Memorial Lecture to the Society for the Study of Fertility, University of Bristol, 1986. 
that a large proportion of patients, whose spermatozoa fail to penetrate oocytes in this assay, will remain unresponsive even when calcium influx is induced by the ionophore, A23187 (Aitken et al., 1984). These results suggest that the defective sperm function identified under such conditions involves lesions, downstream from calcium influx, which prevent the plasma and outer acrosome membranes from engaging in the membrane fusion events associated with fertilization.

This failure to exhibit hamster oocyte penetration in the presence of calcium ionophores is an extremely common phenomenon occurring in $70 \%$ of men exhibiting oligozoospermia (Aitken et al., 1984) and about $40 \%$ of patients possessing varicocoeles (Aitken et al., 1987). The purpose of our current research is to determine the biochemical basis for such failures of membrane fusion. The fact that human spermatozoa are known to be susceptible to lipid peroxidation (Jones et al., 1979) and that rabbit (Holland \& Storey, 1981) and bovine (Tosic \& Walton, 1950) spermatozoa have been shown to generate reactive oxygen species such as hydrogen peroxide, led us to postulate that peroxidative damage, involving the proteins and unsaturated fatty acids present in the sperm plasma membrane, might be involved in the aetiology of defective sperm function. In this study, we present evidence indicating that the human spermatozoon is capable of producing reactive oxygen species and that the loss of sperm function encountered in certain cases of male infertility is associated with the excessive activity of this free oxygen-radical generating system.

\section{Materials and Methods}

Donors. The donors for this research were selected from a panel of 72 normal healthy males all of whom had undergone complete physical examination and had been screened for antibodies against hepatitis B and HTLV III. All donors possessed normal semen profiles with the following characteristics: $>20 \times 10^{6}$ spermatozoa $/ \mathrm{ml} ;>40 \%$ motility and $>40 \%$ normal morphology. In addition, analyses were performed on: (a) 22 patients possessing clinical varicocoeles, in each of whom active refluxing of blood down the left internal spermatic vein had been confirmed with a Doppler stethoscope; (b) 36 patients exhibiting idiopathic infertility, in whom the semen profile for the male partner accorded with the above criteria of normality and the female partner was normal with respect to history and examination, frequency and duration of menstrual cycles, luteal progesterone concentrations and tubal patency as assessed by the passage of dye at laparoscopy (Aitken et al., 1982); (c) 2 cases of isolated oligozoospermia $\left(<20 \times 10^{6}\right.$ spermatozoa/ml); and (d) 2 cases of isolated asthenozoospermia ( $<40 \%$ motility).

Analysis of sperm function. The ability of human spermatozoa to exhibit sperm-oocyte fusion in response to a calcium signal generated by A23187 was assessed using the protocol described by Aitken et al. (1984). In brief, this procedure involved washing each semen sample 3 times, using repeated cycles of centrifugation $(500 \mathrm{~g}$ for $5 \mathrm{~min}$ ) and resuspension in Medium BWW (Biggers et al., 1971). In addition 7 samples were prepared by a swim-up procedure and on discontinuous Percoll gradients (Berger et al., 1985) in order to ensure that the activity recorded was associated with the isolated spermatozoa and not any other cell type present in the semen. The spermatozoa were finally suspended at a concentration of $20 \times 10^{6} / \mathrm{ml}$ and diluted with an equal volume of Medium BWW containing an aqueous suspension of the $\mathrm{Ca}^{2+}, \mathrm{Mg}^{2+}$ salt of $\mathrm{A} 23187\left(0.05 \mathrm{mg} / \mathrm{ml}\right.$ ) (Aitken et al., 1984). After 3-h incubation at $37^{\circ} \mathrm{C}$ in an atmosphere of $5 \% \mathrm{CO}_{2}$ in air, the spermatozoa were centrifuged at $500 \mathrm{~g}$ for $5 \mathrm{~min}$, resuspended in normal Medium BWW and dispersed as $50 \mu \mathrm{l}$ droplets under liquid paraffin. Zona-free hamster oocytes were then added to the sperm suspension and incubated at $37^{\circ} \mathrm{C}$ in $5 \% \mathrm{CO}_{2}$ in air for $3 \mathrm{~h}$ before assessing the percentage of oocytes exhibiting penetration (Aitken et al., 1984). Penetration rates were finally adjusted for small differences in motility between samples, using the Poisson model described previously (Aitken \& Elton, 1986).

Reactive oxygen species. Human spermatozoa were washed free of seminal plasma, as previously described, and finally resuspended at a concentration of $20 \times 10^{6} / \mathrm{ml}$. Then $1 \mu l$ luminol (5-amino-2,3-dihydro-1,4phthalazinedione), stored as a $100 \mathrm{mM}$ stock solution in dimethyl sulphoxide (DMSO), was added to $500 \mu \mathrm{l}$ of spermatozoa followed by the addition of $500 \mu \mathrm{l}$ Medium BWW (control) or an equal volume of Medium BWW containing the $\mathrm{Ca}^{2+}, \mathrm{Mg}^{2+}$ salt of A23187 $(0.05 \mathrm{mg} / \mathrm{ml})$. Luminescence was subsequently recorded on a Berthold, Biolumat LB 9500T luminometer, in the integration mode. Luminol is an extremely sensitive chemiluminescent probe which will react with a variety of reactive oxygen species $\left(\mathrm{H}_{2} \mathrm{O}_{2}, \mathrm{O}_{2}-, \cdot \mathrm{OH},{ }^{1} \mathrm{O}_{2}\right)$. In essence, the cyclic hydrazide is dehydrogenated to the diazaquinone, and the latter is then oxygenated to yield a cyclic peroxide that disintegrates to yield the electronically excited aminophthalate anion. At neutral $\mathrm{pH}$, luminol exhibits a tendency towards hydrophobic binding and is thought to permeate cells giving an indication of reactive oxygen generated within a cell (in a phagolysosome for example) as well as that released into the extracellular space (Allen, 1982). In all the experiments cited, background luminescence was determined before luminol addition and found to be negligible and uninfluenced by exposure to A23187. 


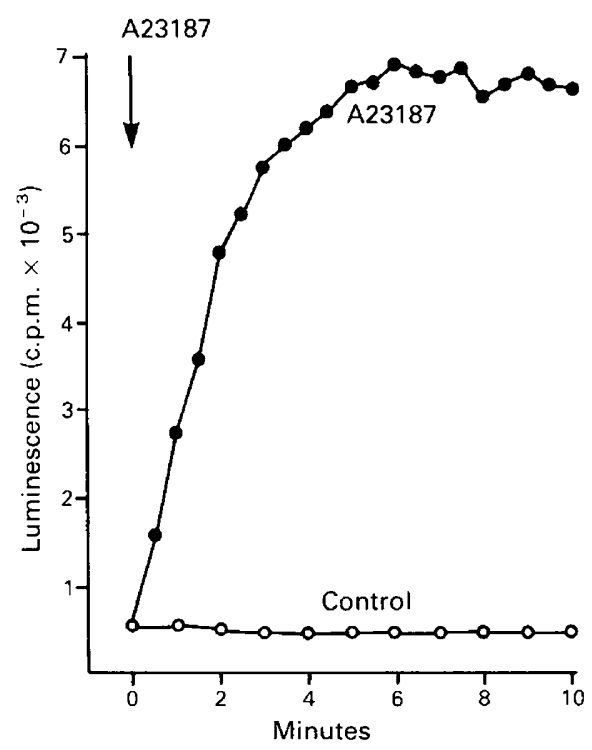

Fig. 1. Production of reactive oxygen species in response to the divalent cation ionophore, A23187.

\section{Results}

Production of reactive oxygen species in response to $A 23187$

Suspensions of normal, functional human spermatozoa produced low levels of reactive oxygen species in their resting state (Figs 1 \& 2). However, upon addition of A23187, a sudden burst of reactive oxygen species production was observed which reached its peak within 3-5 min (Fig. 1). This burst of activity was recorded with human spermatozoa which had been prepared by the conventional centrifugation technique, isolated by the 'swim up' procedure or recovered from Percoll gradients, indicating that the formation of reactive oxygen species is a property of the spermatozoa and not of any other cell type contaminating the seminal plasma.

\section{$\mathrm{Ca}^{2+}$ dependency}

This response to A23187 was dependent upon the presence of high concentrations of extracellular calcium, indicating that it is the ability of A23187 to act as a calcium ionophore, rather than a non-specific perturbance of the sperm plasma membrane (Klausner et al., 1979), which was responsible for the burst of production of reactive oxygen species. Hence when calcium was omitted from Medium BWW (giving a free ionic calcium concentration of about $10 \mu \mathrm{M}$ ) or when this calcium-depleted medium was supplemented with $100 \mu \mathrm{M}$-EGTA (to reduce the free ionic calcium content of the medium to an exceedingly low level) no statistically significant changes in free oxygen radical production were observed upon the addition of A23187 (Table 1).

\section{Influence of mitochondrial inhibitors}

The reactive oxygen species generated in response to A23187 were not of mitochondrial origin. Hence, when the mitochondrial inhibitors oligomycin, antimycin $\mathrm{A}$ and rotenone were added to suspensions of human spermatozoa for $5 \mathrm{~min}$ before the addition of A23187, no changes were 

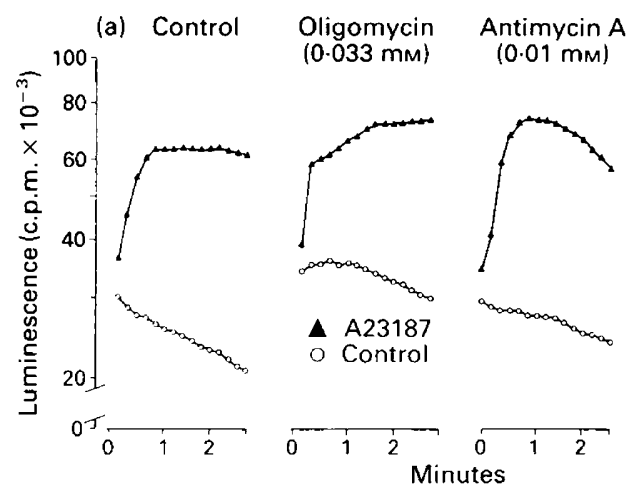

\section{Rotenone \\ $(0.01 \mathrm{~mm})$}
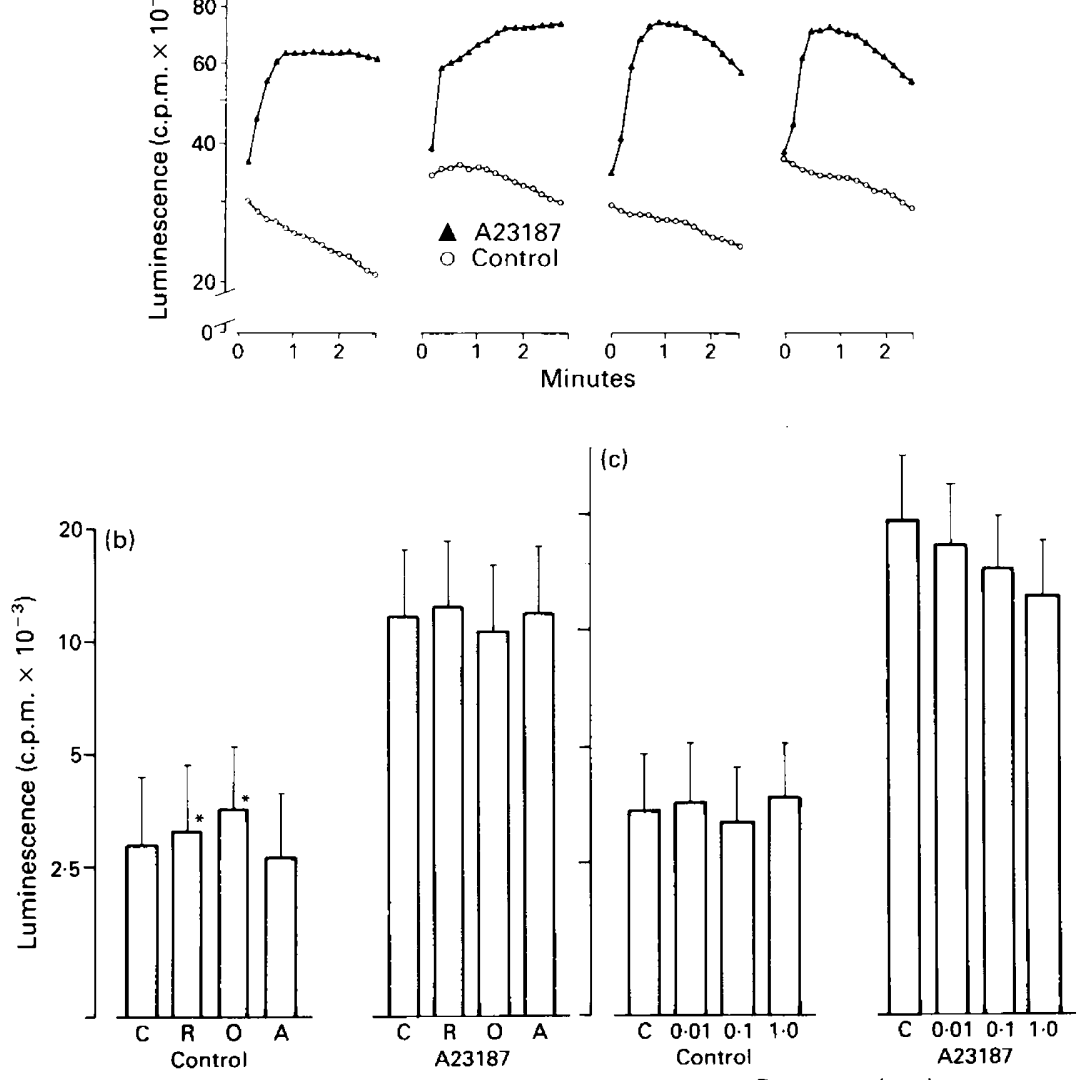

Fig. 2. Effect of preincubation with mitochondrial inhibitors for $5 \mathrm{~min}$ on the generation of reactive oxygen species by human spermatozoa. (a) Longitudinal analysis monitoring production of reactive oxygen species every $10 \mathrm{sec}$ after the addition of A23187; representative example from 3 independent experiments. (b) Changes in production of reactive oxygen species measured at a fixed time interval, $3 \mathrm{~min}$ after the addition of A23187. $\mathrm{C}=$ control, $\mathrm{R}=$ rotenone $(0.01 \mathrm{~mm}), \mathrm{O}=$ oligomycin $(0.033 \mathrm{~mm})$ and $\mathrm{A}=\operatorname{antimycin}(0.01 \mathrm{~mm}) ; n=8$, ${ }^{*} P<0.05$ (Wilcoxon matched-pairs signed-ranks test). (c) Dose-response analysis of the effect of rotenone on production of reactive oxygen species production; $\mathrm{C}=$ control. For (b) and (c) values are mean \pm s.e.m. for 7 observations.

Table 1. Influence of $\mathrm{Ca}^{2+}$ on the generation of reactive oxygen species by human spermatozoa

\begin{tabular}{lccr}
\hline & Medium BWW & No added $\mathrm{Ca}^{2+}$ & \multicolumn{1}{c}{$\mathrm{Ca}^{2+}$ free } \\
\hline Control & $1766 \pm 1123 \cdot 2$ & $1650 \pm 1125$ & $863 \pm 433$ \\
A23187 & $7311 \pm 3642^{* *}$ & $2911 \pm 1836$ & $2215 \pm 1296$ \\
\hline
\end{tabular}

Values indicate photons (c.p.m.) released in the presence of luminol during an integrated $10-\mathrm{sec}$ period, $3 \mathrm{~min}$ after A23187 addition. These results represent the mean \pm s.e.m. of 7 individual samples.

${ }^{* *} P<0.01$, Wilcoxon matched-pairs signed-ranks test. 


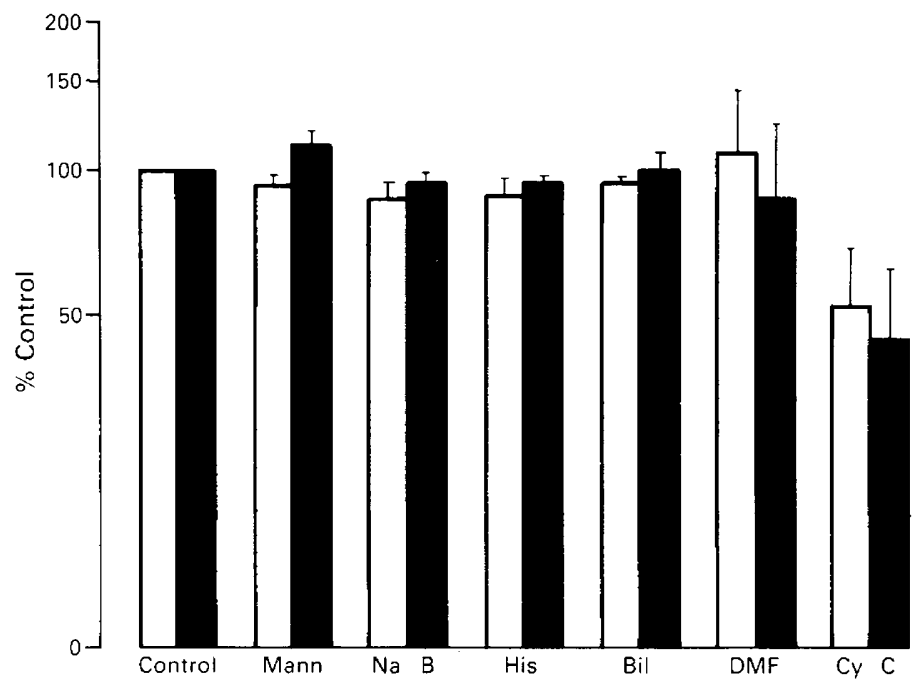

Fig. 3. Influence of selected scavengers on the basal level of reactive oxygen species production (open columns) and the response to A23187 (solid columns). Mann = mannitol; $\mathrm{NaB}=$ sodium benzoate; $\mathrm{His}=$ histidine $\mathrm{HCl} ; \mathrm{Bil}=$ bilirubin; $\mathrm{DMF}=$ dimethyl furan; $\mathrm{CyC}=$ cytochrome $\mathrm{C}$. Values are mean \pm s.e.m. for 3 observations.

observed in the subsequent response to the latter in longitudinal analyses of individual samples (Fig. 2a) or when a cohort of independent samples was assessed at a fixed time point 3 min after A23187 addition (Fig. 2b). Rotenone and oligomycin even caused a significant increase in the basal level of reactive oxygen species production $(P<0 \cdot 05)$ measured before the addition of A23187 (Fig. 2b).

Recent studies on rabbit spermatozoa with the spin label TEMPONE have demonstrated that the most likely site of electron leakage from the mitochondrial electron transport chain is at ubiquinol (Chapman et al., 1985). Since this source of electrons can be effectively blocked by rotenone (Chapman et al., 1985), the studies with this particular inhibitor were extended to include a dose-response analysis (Fig. 2c). The results revealed that with doses as high as $1 \mathrm{~mm}$, rotenone could not influence the ability of A23187 to induce the formation of reactive oxygen species, again indicating an extra mitochondrial source for this activity.

\section{Influence of scavengers}

To generate pertinent information on the forms of reactive oxygen being generated by the human spermatozoon, a variety of scavengers was used in an attempt to quench this activity. The scavengers selected included reagents which are capable of interacting with singlet oxygen (dimethyl furan, bilirubin and histidine $\mathrm{HCl}$ ) or the hydroxyl radical (histidine $\mathrm{HCl}$, sodium benzoate and mannitol) none of which significantly influenced either the basal level of reactive oxygen species production or the response to A23187 (Fig. 3). In contrast, cytochrome C, a scavenger of the superoxide anion radical, reduced the basal level of reactive oxygen species production and the response to A23187 by about 50\% (Fig. 3).

\section{Time- and sample-dependent variation}

To determine whether capacitation of the spermatozoa influenced the pattern of reactive oxygen species production, longitudinal studies were performed on samples from 10 donors 

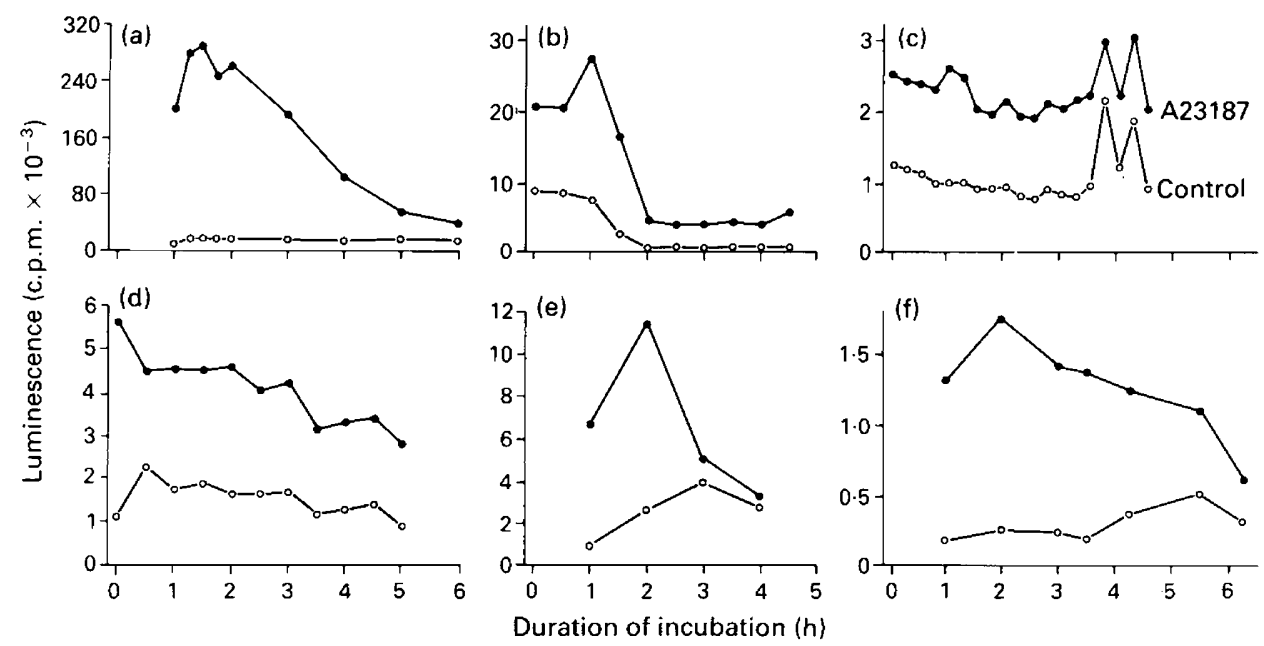

Fig. 4. Changes in generation of reactive oxygen species during prolonged incubation in vitro; (a) (f) represent the patterns obtained with 6 separate individuals. Each point represents the level of production recorded $3 \mathrm{~min}$ after the addition of A23187 or an equivalent volume of Medium BWW, as the control.

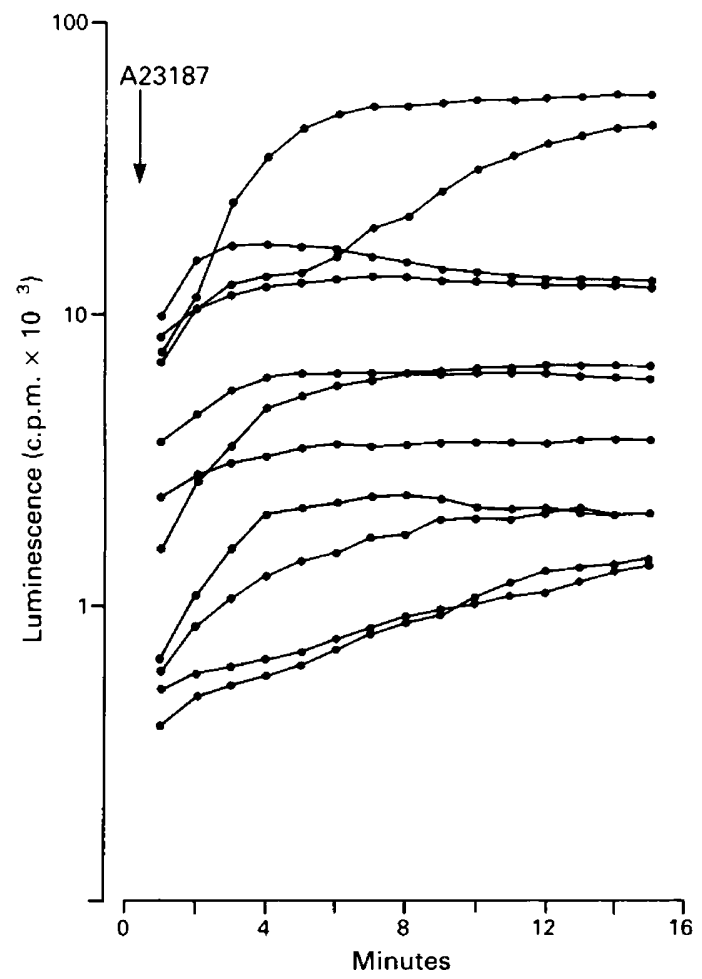

Fig. 5. Time course of the response to A23187 illustrating the inter-individual variation in the production of reactive oxygen species. 


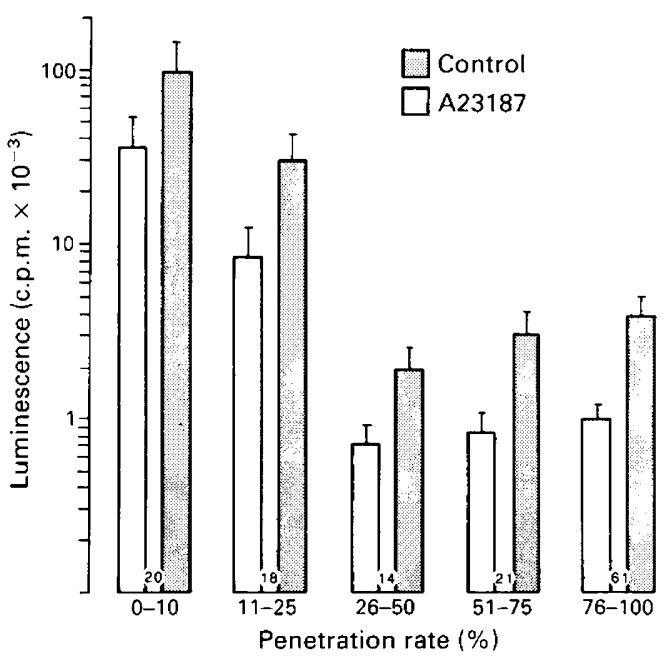

Fig. 6. Relationship between the ability of human spermatozoa for sperm-oocyte fusion and the production of reactive oxygen species. Values are mean \pm s.e.m. for no. of samples indicated at foot of columns.

incubated in Medium BWW for periods of 4.5-6.5 h, depending on the number of cells available for analysis. In a majority of samples $(9 / 10)$ a similar pattern of reactive oxygen species generation was observed, with a peak response to A23187 being recorded within the first $2 \mathrm{~h}$ of incubation, followed by a progressive decay in activity over the remainder of the study period (Fig. 4a, b, d, e, f). The only exception was one sample (Fig. 4c) which exhibited sudden peaks of activity after 3.75 and $4 \cdot 5 \mathrm{~h}$ of incubation in the control and A23187-stimulated samples.

Another feature of these time-dependent studies was the considerable variation between samples in the magnitude of the response to A23187. The sample illustrated in Fig. 4(a), for example, was 100 times more active in generating free oxygen radicals than the samples presented in Figs 4(c) and 4(f). This inter-individual variability is further illustrated in Fig. 5 which depicts the pattern of reactive oxygen species production observed during the first $15 \mathrm{~min}$ after the addition of A23187, in 11 independent samples.

\section{Reactive oxygen species production and sperm-oocyte fusion}

To determine whether the above differences in activity bore any relationship to the relative fertilizing ability of the samples, simultaneous assessments of both these aspects of sperm function were made on 134 separate individuals (Fig. 6). The results indicated that samples exhibiting penetration rates in excess of $25 \%$ were characterized by low levels of reactive oxygen species production which showed a small, 2-3-fold rise in response to the administration of A23187. In contrast, specimens exhibiting a poor capacity for oocyte fusion (11-25\% penetration), and particularly those in which less than $10 \%$ of the oocytes were penetrated after treatment with A23187, were extremely active in the generation of reactive oxygen species (Fig. 6). The mean, basal level of activity exhibited by these defective spermatozoa was 40 -fold higher than that observed in normal functional cells, and was increased still further upon addition of the ionophore.

\section{Discussion}

The results obtained in this study indicate that human spermatozoa are capable of generating reactive oxygen species and that this property may be implicated in the aetiology of male infertility. Reactive oxygen species production is an activity normally associated with the phagocytic activity 
of polymorphonuclear neutrophils and macrophages, the primary purpose of which is to effect the destruction of invading micro-organisms (Babior et al., 1973; Babior, 1978; Klebanoff, 1980). In such cells, the phagocytic process is associated with a sudden increase in oxygen consumption and the genesis of superoxide anion, via mechanisms which can be stimulated by the divalent cation ionophore, A23187 (Pick \& Keisari, 1981). The calcium influx induced by this reagent appears to stimulate superoxide radical generation by a membrane-bound NADPH oxidase system, through the mediation of protein kinase $\mathrm{C}$, a regulatory enzyme which depends on calcium, phosphatidylserine and diacylglycerol, for maximal activity (Gabig \& Babior, 1979; Fujita et al., 1984). The mechanisms by which reactive oxygen species are produced by human spermatozoa may involve similar pathways. A23187 was an effective stimulator of this activity in the presence of extracellular calcium, and the evidence provided by the use of oxygen radical scavengers, suggests that the major product generated by the human spermatozoon is the superoxide anion radical. Furthermore, activators of protein kinase $\mathrm{C}$, such as 1-oleoyl,2-acetyl glycerol or the 12-myristate,13-acetate phorbol ester, are potent stimulators of reactive oxygen species production by the human spermatozoon. A similar NADPH oxidase system even seems to be involved, since NADPH will induce a burst of production of reactive oxygen species production in Triton-permeabilized spermatozoa and isolated membrane pellets. In these circumstances other potential substrates for the production of reactive oxygen species, such as xanthine or the amino acids phenylalanine, tyrosine and tryptophan (Tosic \& Walton, 1950), have no such effect (R. J. Aitken \& J. S. Clarkson, unpublished observations).

Since luminol is a membrane-permeant reagent at neutral $\mathrm{pH}$ it is not possible to state whether the reactive oxygen species are being generated within the spermatozoon or released to the outside. Further experiments comparing the chemiluminescent responses obtained with luminol with those generated by membrane impermeant probes, such as lucigenin, will be required to answer this point. The fact that exogenous superoxide dismutase does not inhibit the luminol response (R. J. Aitken \& J. S. Clarkson, unpublished observations) suggests that the source of the reactive oxygen may be intracellular. This will be an important point to confirm since, if this is the case, defective spermatozoa, generating high levels of reactive oxygen species would be unlikely to damage normal functional cells in the same ejaculate.

The reactive oxygen species generated by human spermatozoa do not seem to have a mitochondrial origin. This is, again, in keeping with similar studies on phagocytic leucocytes, indicating that inhibition of the mitochrondrial respiratory chain has no effect on the generation of superoxide anion or hydrogen peroxide (Reiss \& Roos, 1978). The demonstration that rotenone, at doses as high as $1 \mathrm{~mm}$, could not inhibit the A23187-induced production of reactive oxygen species by human spermatozoa is particularly important, because electron leakage from rabbit sperm mitochondria has been demonstrated using the spin label TEMPONE (Chapman et al., 1985). This activity was decreased by rotenone and increased in the presence of antimycin $\mathrm{A}$ and potassium cyanide, suggesting that the source of electrons was ubiquinol. Since rotenone did not inhibit the basal level of reactive oxygen species production or the response to A23187 by human spermatozoa, a mitochrondrial source of electrons for the production of the superoxide anion radical by human spermatozoa seems unlikely.

The biological significance of the reactive oxygen species produced by human spermatozoa has yet to be elucidated. Time-dependent studies indicated that this activity exhibited spontaneous changes during capacitation, which in most cases resulted in a peak of activity within the 1st or 2nd hour of incubation followed by a progressive decline. Whether this pattern of activity has any bearing on the capacitation process itself is not known. It is possible that the initial activity is simply a consequence of the mechanical perturbation of the sperm plasma membrane induced by the centrifugation procedures employed in preparing the cells. Such perturbation of the plasma membrane of phagocytic leucocytes is known to induce the release of the superoxide anion radical (Babior, 1978) and we have obtained evidence indicating that human spermatozoa can be activated by a similar mechanism (R. J. Aitken \& J. S. Clarkson, unpublished observations). 
A clue to the biological role for this system may be found in another non-phagocytic cell, the adipocyte, which has been reported to possess a membrane-bound NADPH oxidase system capable of generating reactive oxygen species (Mukherjee et al., 1978). In this cell type activation of the NADPH oxidase with insulin results in an alkalinization of the interior of the cell (Mukherjee \& Mukherjee, 1981). Since a rise in intracellular $\mathrm{pH}$ is thought to be involved in the induction of the acrosome reaction (Working \& Meizel, 1983), it is possible that the physiological mechanisms responsible for this event involve the mediation of a superoxide radical-generating system in the sperm plasma membrane. If this were the case, the physiological stimulus for activating this system may well reside in some component of the zona pellucida (Florman et al., 1984; Singer et al., 1985).

Alternatively, generation of reactive oxygen species at the zona surface may lead to oxidative changes in the latter, which may contribute to the block to polyspermy by rendering the zona more resistant to sperm penetration (Schmell \& Gulyas, 1980). Such a mechanism may even involve co-operation between the surplus spermatozoa bound to the zona pellucida and the oocyte, the latter releasing an ovoperoxidase in the cortical granules, which could utilize the hydrogen peroxide produced by the spermatozoa (formed by the dismutation of superoxide anion, through the mediation of superoxide dismutase; Mennella \& Jones, 1980) to catalyse the cross linking of tyrosine residues in the zona matrix.

The clinical significance of the superoxide radical-generating system lies in the inverse relationship recorded between the ability of human spermatozoa to produce reactive oxygen species and their capacity to exhibit sperm-oocyte fusion in response to A23187. Samples exhibiting a normal capacity for oocyte fusion (i.e. more than $25 \%$ of the oocytes penetrated; Aitken et al., 1984) were characterized by a low level of reactive oxygen species production, which exhibited a small, 2-3-fold, rise on exposure to A23187. In contrast, defective spermatozoa with little or no capacity for sperm-oocyte fusion, exhibited a level of reactive oxygen species production which was about 40 times that observed in normal, functional cells. We have not yet had an opportunity to examine the spermatozoa of such patients to determine which particular species of reactive oxygen are being produced in excessive amounts. Superoxide anion radical and, as a result a dismutation, hydrogen peroxide, are the obvious candidates, but this will have to be confirmed experimentally.

Failure to respond to a calcium influx by undergoing sperm-oocyte fusion is a specific defect which has been observed in all classes of infertile men examined to date, including those with oligozoospermia (Aitken et al., 1984), varicocoele and idiopathic infertility (R. J. Aitken \& J. S. Clarkson, unpublished observations). The proportion of patients in each category exhibiting a loss of sperm function in association with the hyperactive production of reactive oxygen species is the subject of current investigation. Results to date indicate that in cases of oligozoospermia $(n=11)$, varicocoele $(n=49)$ and unexplained infertility $(n=76)$ an impaired response to A23187 (penetration rates of $<10 \%$ ) was observed in $54.5 \%, 38.8 \%$ and $31.6 \%$ of patients, respectively. This loss of sperm function was associated with the excessive generation of reactive oxygen species in all $(100 \%)$ of the oligozoospermic patients, $52 \cdot 6 \%$ of the varicocoele cases and $58 \cdot 3 \%$ of men with unexplained infertility.

The hyperactive production of reactive oxygen species may be directly responsible for the loss of sperm function through the peroxidation of unsaturated fatty acids (Jones et al., 1979) or the denaturation of proteins in the sperm plasma membrane. The presence of superoxide dismutase in human seminal plasma and the human spermatozoon (Mennella \& Jones, 1980) suggests that any superoxide radicals produced by these cells would rapidly dismutate to hydrogen peroxide. Since mammalian spermatozoa do not appear to exhibit catalase activity, this hydrogen peroxide may well induce peroxidative damage in the sperm plasma membrane, unless an active glutathione peroxidase system is present. Assessment of the degree of glutathione peroxidase activity in human spermatozoa, and its role in protecting these cells against peroxidative damage, are high priority areas for the future.

An alternative possibility is that the excessive generation of reactive oxygen species may be a consequence (rather than a cause) of a primary defect in the differentiation of the sperm plasma 
membrane, such that the control mechanisms regulating this activity are no longer effective. Elucidating the relationship between superoxide radical generation and the loss of sperm function may be of significance in the treatment of male infertility, since it raises the possibility of using antioxidants for therapeutic purposes. In terms of fundamental andrology, these studies have provided evidence for a novel superoxide radical-generating system in the human spermatozoon and, for the first time, identified a biochemical lesion in these cells, which appears to be implicated in the aetiology of male infertility.

We thank Mr D. W. Richardson and Dr D. S. Irvine for recruiting and screening the donors used in this research project.

\section{References}

Aitken, R.J. (ed.) (1986) The zona-free hamster oocyte penetration test and the diagnosis of male infertility. Int. J. Androl. Suppl. 6.

Aitken, R.J. \& Elton, R.A. (1986) Quantitative analysis of sperm-egg interaction in the zona-free hamster penetration test. Int. J. Androl. Suppl. 6, 14-30.

Aitken, R.J., Best, F.S.M., Richardson, D.W. Djahanbakhch, O., Mortimer, D., Templeton, A.A. \& Lees, M.M. (1982) An analysis of sperm function in cases of unexplained infertility: conventional criteria, movement characteristics and fertilizing capacity. Fert. Steril. 38, 212-221.

Aitken, R.J., Ross, A., Hargreave, T., Richardson, D.W. \& Best, F.S.M. (1984) Analysis of human sperm function following exposure to the ionophore A23187. $J$. Androl. 5, 321-329.

Aitken, R.J., Clarkson, J.S., Huang, G-F. \& Irvine, D.S. (1987) Cell biology of defective sperm function. In Recent Advances in Spermatology. Ed. H. Mohri. Japanese Scientific Societies Press, Tokyo (in press).

Allen, R.C. (1982) Biochemiexcitation: chemiluminescence and the study of biological oxygenation reactions. In Chemical and Biological Generation of Excited States, pp. 310-341. Eds W. Adam \& G. Cilento. Academic Press, London.

Babior, B.M. (1978) Oxygen-dependent microbial killing by phagocytes. New Engl. J. Med. 298, 659-668.

Babior, B.M., Kipnes, R.S. \& Curnutte, J.T. (1973) The production by leucocytes of superoxide, a potential bactericidal agent. J. clin. Invest. 52, 741-744.

Berger, T., Marrs, R.P. \& Moyer, D.L. (1985) Comparison of techniques for selection of motile spermatozoa. Fert. Steril. 43, 268-273.

Biggers, J.D., Whitten, W.K. \& Whittingham, D.G. (1971) The culture of mouse embryos in vitro. In Methods of Mammalian Embryology, pp. 86-116. Ed. J. C. Daniel, Jr. Freeman, San Francisco.

Chapman, D.A., Killian, G.J., Gelerinter, E. \& Jarrett, M.T. (1985) Reduction of the spin-label TEMPONE by ubiquinol in the electron transport chain of intact rabbit spermatozoa. Biol, Reprod. 32, 884-893.

Florman, H.M., Bechtol, K.B. \& Wassarman, P.M. (1984) Enzymatic dissection of the functions of the mouse egg's receptor for sperm. Devl Biol. 106, 243-255.

Fujita, I., Irita, K., Takeshige, K. \& Minakami, S. (1984)
Diacylglycerol 1-oleoyl-2-acetyl-glycerol, stimulates superoxide-generation from human neutrophils. Biochem. Biophys. Res. Commun. 120, 318-324.

Gabig, T.G. \& Babior, B.M. (1979) The $\mathrm{O}_{2}^{-}$-forming oxidase responsible for the respiratory burst in human neutrophils. J. Biol. Chem. 254, 90709074.

Holland, M.K. \& Storey, B.T. (1981) Oxygen metabolism of mammalian spermatozoa. Biochem. J. 198, 273-280.

Hull, M.G.R. (1986) Infertility: nature and extent of the problem. In: Human Embryo Research; Yes or No, pp. 24-38. Eds G. Bock \& M. O'Connor. Ciba Foundation. Tavistock Publications, London.

Jones, R., Mann, T. \& Sherins, R. (1979) Peroxidative breakdown of phospholipids by human spermatozoa, spermicidal properties of fatty acid peroxides, and protective action of seminal plasma. Fert. Steril. 31, 531-537.

Klausner, R.D., Fishmen, M.C. \& Karnovsky, M.J. (1979) Ionophore A23187 disrupts membrane structure by modifying protein-lipid interactions. Nature, Lond. 281, 82-83.

Klebanoff, S.J. (1980) Oxygen metabolism and the toxic properties of phagocytes. Annls Int. Med. 93, 480-489.

Mennella, M.R.T. \& Jones, R. (1980) Properties of spermatozoal superoxide dismutase and lack of involvement of superoxides in metal-ion-catalysed lipid-peroxidation reactions in semen. Biochem. J. 191, 289-297.

Mukherjee, S.P. \& Mukherjee, C. (1981) Metabolic activation of adipocytes by insulin accompanied by an early increase in intracellular pH. Ann. N.Y. Acad. Sci. 372, 347-351.

Mukherjee, S.P., Lane, R.H. \& Lynn, W.S. (1978) Endogenous hydrogen peroxide and peroxidative metabolism in adipocytes in response to insulin and sulfhydryl reagents. Biochem. Pharmacol. 27, 2589-2594.

Pick, E. \& Keisari, Y. (1981) Superoxide anion and hydrogen peroxide production by chemically elicited peritoneal macrophages-induction by multiple nonphagocytic stimuli. Cell. Immunol. 39, 301-318.

Reiss, M. \& Roos, D. (1978) Differences in oxygen metabolism of phagocytosing monocytes and neutrophils. J. clin. Invest. 61, 480-488. 
Schmell, E.D. \& Gulyas, B.J. (1980) Ovoperoxidase activity in ionophore treated mouse eggs. II. Evidence for the enzyme's role in hardening the zona pellucida. Gamete Res. 3, 279-290.

Singer, S.L., Lambert, H., Overstreet, J.W., Hanson, F.W. \& Yanagimachi, R. (1985) The kinetics of human sperm binding to human zona pellucida and zona-free hamster oocyte in vitro. Gamete Res. 12, 29-39.

Tosic, J. \& Walton, A. (1950) Metabolism of spermatozoa. The formation and elimination of hydrogen peroxide by spermatozoa and effects on motility and survival. Biochem. J. 47, 199-212.
Working, P.K. \& Meizel, S. (1983) Correlation of increased intraacrosomal $\mathrm{pH}$ with the hamster sperm acrosome reaction. J. exp. Zool. 227, 97-107.

Yanagimachi, R., Yanagimachi, H. \& Rogers, B.J. (1976)

The use of zona free animal ova as a test system for the assessment of the fertilizing capacity of human spermatozoa. Biol. Reprod. 15, 471-476.

Received 12 March 1987 